CONFORMAL GEOMETRY AND DYNAMICS

An Electronic Journal of the American Mathematical Society

Volume 10, Pages 21-40 (March 1, 2006)

S $1088-4173(06) 00111-1$

\title{
THE EXISTENCE OF QUASIMEROMORPHIC MAPPINGS IN DIMENSION 3
}

\author{
EMIL SAUCAN
}

For Meir, who insisted

\begin{abstract}
We prove that a Kleinian group $G$ acting on $\mathbb{H}^{3}$ admits a nonconstant $G$-automorphic function, even if it has torsion elements, provided that the orders of the elliptic elements are uniformly bounded. This is accomplished by developing a method for meshing distinct fat triangulations which is fatness preserving. We further show how to adapt the proof to higher dimensions.
\end{abstract}

\section{INTRODUCTION}

In this article we study the existence of $G$-automorphic quasimeromorphic mappings (in the sense of Martio and Srebro-see [MS1]) $f: \mathbb{H}^{n} \rightarrow \widehat{\mathbb{R}^{n}}, \widehat{\mathbb{R}^{n}}=\mathbb{R}^{n} \cup\{\infty\}$; i.e. such that

$$
f(g(x))=f(x) \text {, for any } x \in \mathbb{H}^{n} \text { and for all } g \in G \text {; }
$$

where $G$ is a Kleinian group acting on $\mathbb{H}^{n}$, i.e. a discontinuous group of orientation preserving isometries of $\mathbb{H}^{n}$.

Our principal goal is to prove the following:

Theorem 1.1. Let $G$ be a Kleinian group with torsion acting on $\mathbb{H}^{n}, n \geq 3$. If the elliptic (i.e. torsion) elements of $G$ have uniformly bounded orders, then there exists a non-constant G-automorphic quasimeromorphic mapping $f: \mathbb{H}^{n} \rightarrow \widehat{\mathbb{R}^{n}}$.

The importance of Theorem 1.1 above resides partly in the applications of automorphic quasimeromorphic mappings, most notably to the construction of Lattès type uniformly quasiregular mappings (see $[\mathrm{My}]$ ).

In this paper we restrict ourselves to the proof of the theorem in the classical case (i.e. $n=3$ ) only. This restriction is motivated by two reasons:

(a) The proof in the 3-dimensional case employs mainly elementary tools.

(b) It develops and uses a technique for meshing distinct fat triangulations while preserving fatness.

This technique is relevant in Computational Geometry and Mathematical Biology (see [SA]). The same basic method of proof is employed in [S1] to prove the general case, however another method of fattening triangulations is being employed. The existence of quasimeromorphic mappings in any dimension also follows from

Received by the editors December 1, 2003 and, in revised form, January 20, 2006.

2000 Mathematics Subject Classification. 30C65, 57R05, 57M60.

Key words and phrases. Automorphic quasimeromorphic mapping, fat triangulation.

(C)2006 American Mathematical Society Reverts to public domain 28 years from publication 
a more general result concerning the existence of fat triangulations for manifolds with boundary (see [S2]).

The question of whether quasimeromorphic mappings exist was originally posed by Martio and Srebro in MS1; subsequently in MS2 they proved the existence of the above-mentioned mappings in the case of finite co-volume groups, i.e. groups such that $\operatorname{Vol}_{\text {hyp }}\left(\mathbb{H}^{n} / G\right)<\infty$. Also, it was later proved by Tukia ( $[\mathrm{Tu}]$ ) that the existence of non-constant quasimeromorphic mappings (or qm-maps) is assured in the case when $\mathrm{G}$ acts torsionfree on $\mathbb{H}^{n}$. Moreover, since for torsionfree Kleinian groups $G, \mathbb{H}^{n} / G$ is an (analytic) manifold, the next natural question to ask is whether there exist $q m$-maps $f: M^{n} \rightarrow \widehat{\mathbb{R}^{n}}$; where $M^{n}$ is an orientable $n$-manifold. The affirmative answer to this question is due to K. Peltonen (see [Pe]); to be more precise she proved the existence of $q m$-maps in the case when $M^{n}$ is a connected, orientable $\mathcal{C}^{\infty}$-Riemannian manifold.

In contrast with the above results it was proved by Srebro ( $\underline{\mathrm{Sr}}])$ that, for any $n \geq 3$, there exists a Kleinian group $G \triangleright<\mathbb{H}^{n}$ such that there exists no nonconstant, $G$-automorphic function $f: \mathbb{H}^{n} \rightarrow \mathbb{R}^{n}$. More precisely, if $G$ (as above) contains elliptics of unbounded orders with non-degenerate fixed set, then $G$ admits no non-constant $G$-automorphic $q m$-mappings.

All the existence results above were obtained in a constructive manner by using the classical Alexander trick (see [A] ) and in our proof we shall apply the same method.

A uniform bound for the dilatations can be attained (see [MS2, Tu]) if the triangulation considered in the Alexander trick is fat, where fat triangulations are defined as follows:

Definition 1.2. A $k$-simplex $\tau \subset \mathbb{R}^{n}$ (or $\mathbb{H}^{n}$ ); $2 \leq k \leq n$ is $f$-fat if there exists $f \geq 0$ such that the ratio $\frac{r}{R} \geq f$; where $r$ denotes the radius of the inscribed sphere of $\tau$ (inradius) and $R$ denotes the radius of the circumscribed sphere of $\tau$ (circumradius). A triangulation of a submanifold of $\mathbb{R}^{n}$ (or $\left.\mathbb{H}^{n}\right) \mathcal{T}=\left\{\sigma_{i}\right\}_{i \in \mathbf{I}}$ is $f$-fat if all its simplices are $f$-fat. A triangulation $\mathcal{T}=\left\{\sigma_{i}\right\}_{i \in \mathbf{I}}$ is fat if there exists $f \geq 0$ such that all its simplices are $f$-fat; $\forall i \in \mathbf{I}$.

Remark 1.3. The definition above is introduced in $[\mathrm{Pe}$. For equivalent definitions of fatness, see [Ca1], Ca2], CMS, [Mun, and [Tu].

Remark 1.4. Fat triangles are precisely those for which the individual simplices considered in the Alexander method may each be mapped onto a standard $n$-simplex, by an $L$-bilipschitz map, followed by a homotety, with a fixed $L$.

The main steps in the proof of Theorem 1.1 are as follows: Based upon the geometry of the elliptic transformations, construct a fat triangulation $\mathcal{T}_{1}$ of $N_{e}^{*}$, where $N_{e}^{*}$ is a certain closed neighbourhood of the singular set of $\mathbb{H}^{3} / G$. Since $M_{p}=\left(\mathbb{H}^{3} \backslash\right.$ Fix $\left.(G)\right) / G, \operatorname{Fix}(G)=\left\{x \in \mathbb{H}^{3} \mid \exists g \in G \backslash\{I d\}, g(x)=x\right\}$ is an orientable analytic manifold, we can apply Peltonen's result to gain a triangulation $\mathcal{T}_{2}$ of $M_{p}$. Therefore, if the triangulations $\mathcal{T}_{1}$ and $\mathcal{T}_{2}$ are chosen properly, each of them will induce a triangulation of $N_{e}^{*} \backslash N_{e}^{* \prime}$, for a certain $N_{e}^{* \prime} \subsetneq N_{e}^{*}$ (see Section 2).

'Mash' $\mathcal{T}_{1}$ and $\mathcal{T}_{2}$ (in $N_{e}^{*} \backslash N_{e}^{* \prime}$ ), i.e. ensure that the given triangulations intersect into a new triangulation $\mathcal{T}_{0}$ (see Mun, Theorem 10.4). Modify $\mathcal{T}_{0}$ to receive a new chessboard, fat triangulation $\mathcal{T}$ of $\mathbb{H}^{3} / G$. Recall that a triangulation (of an $n$ manifold) is called a chessboard triangulation iff its simplices satisfy the condition that every $(n-1)$-face is incident to an even number of $n$-simplices. 
Apply Alexander's trick to receive a quasimeromorphic mapping $f: \mathbb{H}^{3} / G \rightarrow \widehat{\mathbb{R}^{3}}$. The lift $\widetilde{f}$ of $f$ to $\mathbb{H}^{3}$ represents the required $G$-automorphic quasimeromorphic mapping.

This paper is organized as follows: In Section 2 we present the necessary background on elliptic transformations and we show how to choose and triangulate the closed neighbourhood $N_{e}^{*}$ of the singular set of $\mathbb{H}^{3} / G$, and how to select the 'intermediate zone' where the two different triangulations overlap. Section 3 is dedicated to the main task of fattening the common triangulation. In Section 4 we show how to apply the main result in the construction of a $G$-automorphic quasimeromorphic mapping from $\mathbb{H}^{3}$ to $\widehat{\mathbb{R}}^{3}$. Finally, in Section 5 we indicate how to adapt our construction to higher dimensions.

\section{Constructing and intersecting triangulations}

2.1. Elliptic transformations. Let us first recall the basic definitions and notation. An orientation preserving transformation $f \in I \operatorname{som}\left(\mathbb{H}^{n}\right), f \neq I d$ is called elliptic iff $f$ has a fixed point in $\mathbb{H}^{n}$. If $G$ is a Kleinian group and if $f \in G, f \neq I d$ is an elliptic transformation, then there exists $m \geq 2$ such that $f^{m}=I d$. The smallest $m$ satisfying this condition is called the order of $f$, and it is denoted by $\operatorname{ord}(f)$. In the 3-dimensional case, the fixed point set of $f$, i.e. Fix $(f)=\left\{x \in \mathbb{H}^{n} \mid f(x)=x\right\}$, is a hyperbolic line and will be denoted by $A(f)$ - the axis of $f$. If $A$ is an axis of an elliptic of order $m$, then $A$ is called an $m$-axis.

Remark 2.1. Given any finitely generated Kleinian group $G$ acting on $\mathbb{H}^{3}$ the number of conjugacy classes of elliptic elements of $G$ is finite (see [FM], S3]). Thus Theorem 1.1. holds for finitely generated Kleinian groups acting on $\mathbb{H}^{3}$. However, the result above does not hold for Kleinian groups acting on $\mathbb{H}^{n}, n \geq 4$ (for counterexamples, see [FM], $[\mathrm{KP}]$, $\mathrm{KP} 1$, and $[\mathrm{H}]$ ).

Recall that every discontinuous group is discrete ([Ms], Proposition C.3). Therefore, if the Kleinian group $G$ acts on $\mathbb{H}^{3}$, there are no accumulation points of the elliptic axes in $\mathbb{H}^{3}$. Moreover, if $G$ contains no elliptics with intersecting axes, and if $G$ contains no order two elliptics, then the distances between the axes are bounded from below. To be more precise, the following holds:

Theorem 2.2 (GM1). Let $G$ be a discrete group $G$ acting on $\mathbb{H}^{3}$, and let $f, g \in G$ be such that ord $(f) \geq 3$ or ord $(g) \geq 3$; and such that $A(f) \cap A(g)=\emptyset$. Then there exists $\delta>0$, that is independent of $G, f, g$ such that:

$$
\operatorname{dist}_{\text {hyp }}(A(f), A(g)) \geq \delta
$$

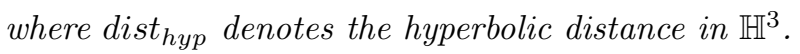

Remark 2.3. The constant $\delta$ above allows one to control the fatness of the triangulation of $N_{e}^{*}$ (see Section 2.2. below).

Remark 2.4. It is important to notice that the result above does not include the case when all the elliptic transformations of $G$ are of order 2. Indeed, examples of discrete groups of isometries of hyperbolic 3 -space can be constructed, such that the distances between the axes of the order 2 elliptics are not bounded from below (see [S3]).

In the presence of nodes, i.e. intersections of elliptic axes, the situation is more complicated. Indeed, the actual computation of the distances between node points 
has been achieved only relatively recently. (See GM1, GM2, and GMMR. See also [DM], Med], and [S3] for a different approach.)

Recall that if $G$ is a Kleinian group, $G$ is countable ( $[\mathrm{Ms}$, Corollary B.4). It follows that the set of elliptics and the set of connected components of Fix $(G)$ are also countable. We denote these sets by $\left\{f_{i}\right\}_{i \geq 1}$ and $\left\{C_{j}\right\}$, respectively.

Moreover, since $G$ is discrete, it follows that the sets $\mathcal{A}=\left\{A_{f_{i}}\right\}_{i \geq 0}$-and hence $\mathcal{S}=\left\{C_{j}\right\}$-have no accumulation points in $\mathbb{B}^{n}$.

We can therefore choose disjoint, $G$-invariant neighbourhoods $N_{j}$ and $N_{j}^{\prime}$ of $C_{j}, N_{j}^{\prime} \subsetneq N_{j}$. Indeed, first choose a neighbourhood $N_{1}$ of $C_{1}$, such that $\bar{N}_{1} \cap$ $\bigcup_{j \geq 2} C_{j}=\emptyset$; then recursively build a neighbourhood $N_{k}$ of $C_{k}$, such that $N_{k} \subset \mathbb{H}^{3} \backslash$ $\left(N_{1} \cup \cdots \cup N_{k-1}\right)$ and $\bar{N}_{k} \cap \bigcup_{j>k} C_{j}=\emptyset$, for all $k \geq 2$. Denote $N_{e}=\bigcup_{j \in \mathbb{N}} N_{j}$, $N_{e}^{\prime}=\bigcup_{j \in \mathbb{N}} N_{j}^{\prime}$. Define $N_{e}^{*}=\left(\bar{N}_{e} \cap \mathbb{H}^{3}\right) / G, N_{e}^{* \prime}=\left(\bar{N}_{e}^{\prime} \cap \mathbb{H}^{3}\right) / G$ (see Figure 1).

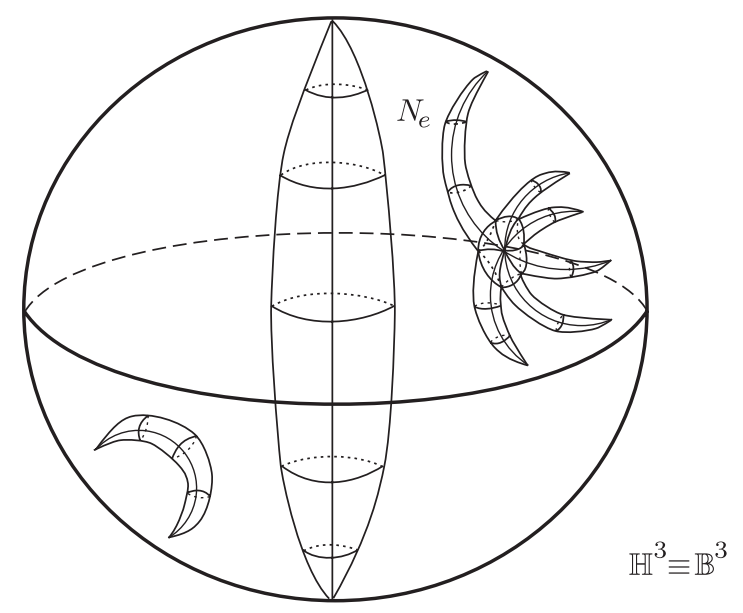

Figure 1.

2.2. Geometric neigbourhoods. If there are no elliptics with intersecting axes, a standard choice for a regular neighbourhood of an $m$-axis will be- for obvious geometric reasons - a doubly-infinite regular hyperbolic $m$-prism (henceforth called a geometric neighbourhood), and a fundamental domain will be a prismatic 'slice', i.e. a fundamental region for the action of $C_{m}$ on $\{m\} \times A(f)$, where $\{m\}$ denotes the regular hyperbolic polygon with $m$ sides and $C_{m}$ denotes the cyclic group of order $m$, i.e. the rotation group of $\{m\}$. In order to triangulate the geometric neighbourhood, we divide it into radial strata of width $\varrho$, for a properly chosen $\varrho>0$, and further partition it into 'slabs' of height $h$. Each prismatic fundamental region thus obtained naturally decomposes into three congruent tetrahedra, generating a $C_{m}$-invariant triangulation of the geometric neigbourhood. (See Figure 2 for an Euclidean (local) representation in the case $m=4$.) Note that, in the special case when the minimal distance $\delta$ between elliptic axes is attained (e.g., if $G$ contains no order two elliptics - see Theorem 2.2), we can choose $\varrho=\delta / \kappa_{0}$, for a certain positive integer $\kappa_{0}$. The fatness of the triangulation of the geometric neighbourhood thus depends, in this case, solely upon 
$\delta, \kappa_{0}$ and $h$, enabling one to control the initial fatness of the geometric triangulation by means of the parameters $\varrho$ and $h$. Moreover, in this case a natural choice for $N_{j}$ and $N_{j}^{\prime}$ is: $N_{j}=N_{j, 1 / 4}=\left\{x \in \mathbb{H}^{3} \mid \operatorname{dist}_{h y p}\left(A_{j}, x\right)<\delta / 4\right\}$ and $N_{j}^{\prime}=N_{j, 3 / 16}=\left\{x \in \mathbb{H}^{3} \mid \operatorname{dist}_{h y p}\left(A_{j}, x\right)<3 \delta / 16\right\}$. In the case when there exist intersecting elliptic axes, instead of $\delta$ one has to consider $\delta^{*}=\min \left(\delta, \delta_{0}\right)$, where $\delta_{0}$ represents the minimal distance between node-points.

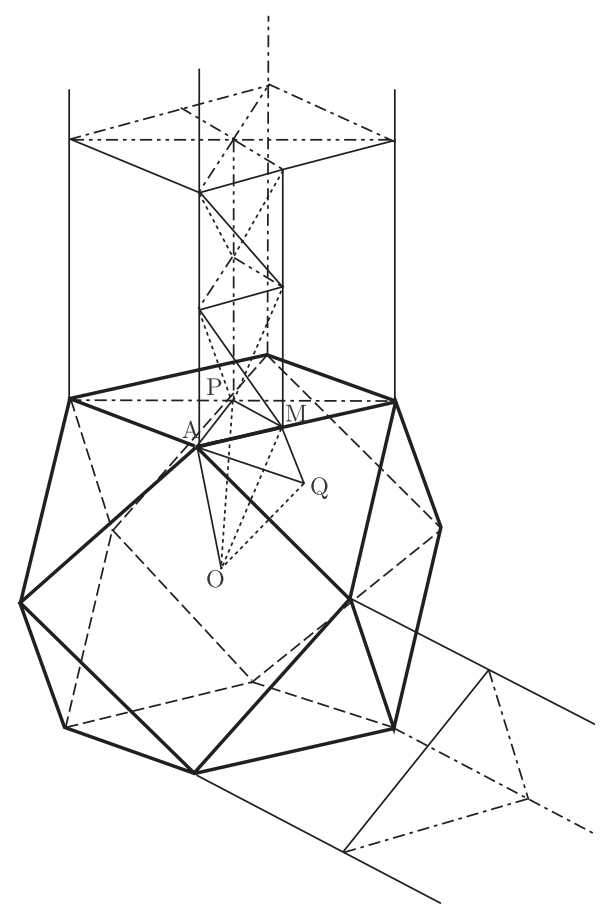

FIGURE 2 .

As geometric neigbourhoods of the node points, the natural choice is that of an Archimedean solid which is a natural carrier of the symmetry group of the desired type: $D_{n}, T, O$ or $I$ (or rather for its spherical counterpart-see [Cox]). See Figure 2 for an Euclidean representation of the geometric neighbourhood $N(p)$ of a node point and some of the fundamental tetrahedra for the action of its stabilizer $\operatorname{Stab}(N(p))$.

\section{Fattening triangulations}

3.1. Preliminaries. We have thus reduced the original problem to that of 'fattening' the intersection of two 3-dimensional finite, fat Euclidean triangulations.

We do this piecemeal, first fattening the 2 -simplices, then the 3 -simplices. It is natural to do so, for the following holds:

Lemma 3.1 ( $[\mathrm{Pe}])$. If an $n$-dimensional simplex is fat, then all its $k$-dimensional faces, $2 \leq k \leq n-1$ are fat.

In particular, in order for an $n$-dimensional simplex to be fat, its 2 -simplices have to be fat. Note that for triangles, with angles $\alpha, \beta, \gamma$, and $r$ and $R$ as above, the 
conditions, $r / R \geq f$ and $\min \{\alpha, \beta, \gamma\} \geq \varphi$, where $\varphi=\varphi(f)$ is an angle depending on $f$, are equivalent. Thus we start fattening 2 -simplices, by ensuring that

$$
\min _{i}\left\{\alpha_{i}, \beta_{i}, \gamma_{i}\right\} \geq \frac{\varphi_{0}}{10},
$$

where the minimum is taken over all the triangles of the resulting triangulation, and where $\varphi_{0}$ is the minimal angle of the original triangulations - ensured by their uniform fatness.

From now on, let $\mathcal{S}=\left\{s_{i}\right\}_{i \in I}$ and $\Sigma=\left\{\sigma_{j}\right\}_{j \in J}$ stand for the simplices of $\mathcal{T}_{1}$ and $\mathcal{T}_{2}$, respectively.

Since the intersections of tetrahedra can be rather unruly, we simplify the situation by requiring that the simplices of one of the triangulations be much smaller than those of the other:

$$
\text { diam } s_{i} \leq \frac{1}{10^{k_{0}}} \operatorname{diam} \sigma_{j} \text {, for any } i \in I \text { and for any } j \in J
$$

where $k_{0}$ is to be determined later.

By using general position arguments (see $[\mathrm{Hu}$, , Mun] , the relative positions of the $s_{i} \mathrm{~s}$ and the $\sigma_{j} \mathrm{~s}$ are now reduced to the following relevant possibilities:

(a) $s_{i} \subset$ int $\sigma_{j}$, or

(a') $s_{i} \subset \operatorname{ext} \sigma_{j}$.

(b) There exists $\eta_{j l}$ such that $\eta_{j l} \cap$ int $s_{i} \neq \emptyset$, where $\eta_{j l}$ is an edge of $\sigma_{j}$, but does not exist $v_{i m}$ in $\eta_{j l}$, where $v_{i m}$ is a vertex of $s_{i}$.

(c) There exists $\nu_{j k}$ such that $\nu_{j k} \in$ int $s_{i}$, where $\nu_{j k}$ is a vertex of $\sigma_{j}$.

(d) int $s_{i} \cap i n t \sigma_{j} \neq \emptyset$, but we are not in one of the previous cases.

3.2. Fattening 2-dimensional triangulations. We start our triangulation fattening process by dealing with the 2-dimensional case first.

Let $\sigma_{j_{0}} \in \Sigma$ be such that there exists a regular neighbourhood $N_{j_{0}}$ of $\sigma_{j}$, triangulated by elements of $\mathcal{S}=\left\{\sigma_{i}\right\}$. Now $\mathcal{S}$ is partitioned by $\sigma_{j}$ into three disjoint families $\mathcal{S}_{0,1}, \mathcal{S}_{0,2}, \mathcal{S}_{0,3}$, where

$$
\begin{aligned}
& \mathcal{S}_{0,1}=\left\{s_{i} \in \Sigma \mid s_{i} \subset \operatorname{int}\left(\sigma_{j_{0}}\right) \text { or } s_{i} \subset \operatorname{int}\left(\sigma_{j_{0}}\right)\right\}, \\
& \mathcal{S}_{0,2}=\left\{s_{i} \in \Sigma \mid \exists v_{0, k} \text {-vertex of } \sigma_{j_{0}} \text { such that } v_{0, k} \in \operatorname{int}\left(s_{i}\right)\right\}, \\
& \mathcal{S}_{0,3}=\left\{s_{i} \in \Sigma \mid \exists ! e_{0, l} \text { - edge of } \sigma_{j_{0}} \text { such that } e_{0, l} \cap \operatorname{int} s_{i} \neq \emptyset\right\} .
\end{aligned}
$$

It is easy to assure - by eventual further subdivision and $\varepsilon$-moves (see e.g. Mun ) - that $\mathcal{S}_{0,3} \cap \mathcal{S}_{1,3}=\emptyset$; where $\mathcal{S}_{1,3}$ is the family corresponding to $\mathcal{S}_{0,3}$, induced by $\sigma_{j_{1}}$, that is adjacent to $\sigma_{j_{0}}$.

The intersections belonging to the family $\mathcal{S}_{0,2}$ are the principal generators of 'un-fatness', for $\measuredangle\left(e_{0, l}, \epsilon_{i, m}\right)$ may be arbitrarily small, where $\epsilon_{i, m} ; m=1,2,3$ are the edges of $s_{i}$ (see Figure 2).

Let $\nu_{0, p}, \nu_{0, m}, \nu_{0, n}$ be three consecutive intersection points of $e_{0, l}$ with edges of two adjacent simplices $s_{i}, s_{i}^{\prime}$ and let $\phi_{0, p}, \phi_{0, m}, \phi_{0, n}$ denote the resulting angles (we always choose the acute angle) (see Figure 3). Now it is not possible that two consecutive angles (out) of the set $\left\{\phi_{0, p}, \phi_{0, m}, \phi_{0, n}\right\}$ are smaller than $\phi_{0}$. Indeed, let us suppose that both $\phi_{0, m}<\phi_{0}$ and $\phi_{0, n}<\phi_{0}$. Then $\phi_{0,1}>\pi-2 \phi_{0,2}$, so $\phi_{0,2}+\phi_{0,3}<2 \phi_{0}$, and thus either $\phi_{0,2}<\phi_{0}$ or $\phi_{0,3}<\phi_{0}$, in contradiction to the fatness of $s_{i}$. 


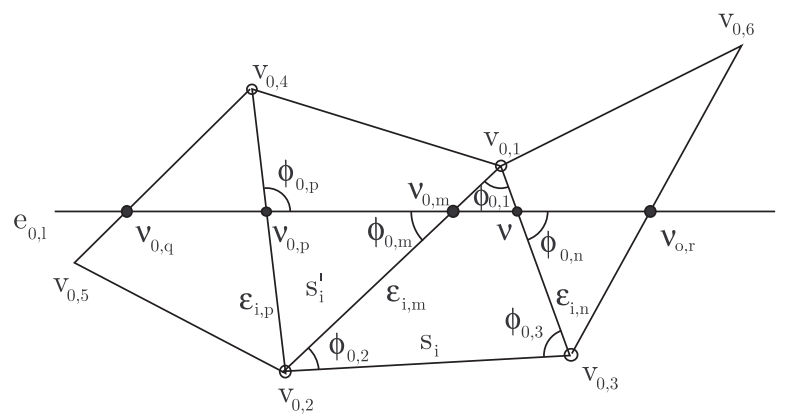

FiguRE 3 .

The fact above implies that we obtain two quadrilaterals which contain the 'bad' points $\nu_{0, p}$ and $\nu_{0, n}$ in their (respective) interiors; let them be

$$
Q_{1}=\square \nu_{0,1} \nu_{0,4} \nu_{0,5} \nu_{0,2}
$$

and

$$
Q_{1}=\square \nu_{0,2} \nu_{0,3} \nu_{0,6} \nu_{0,1}
$$

We erase the segments $\nu_{0, q} \nu_{0, p}, \nu_{0,2} \nu_{0, p}, \ldots, \nu_{0, n} \nu_{0, r}$ and we replace them with segments that will 'fattily' triangulate the quadrilaterals in question. These triangles have 'big' angles (since their angles belong to fat triangles or are the sum of two such angles).

We distinguish between two cases: (a) $Q_{i}$ is convex, and (b) $Q_{i}$ is not convex. We first take care of the simpler case.

(a) Let $Q$ be a convex quadrangle such that all its angles are $\geq \phi_{0}$ and let $\vec{l}_{i, k}$ be the ray interior to $\measuredangle A_{i-1} A_{i} A_{i+1}$ such that $\measuredangle \vec{l}_{i, k} A_{i} A_{k}=\frac{1}{4} \alpha_{i} ; i=0, \ldots, 3 ; k \in$ $\{i-1, i+1\}$ are considered $\bmod 4$, of course. (See Figure 4 , where $Q^{*}=\chi_{1} \ldots \chi_{9}$.) Then the rays $\left\{\vec{l}_{i, k}\right\}_{i, k}$ generate a convex polygon $Q^{*} \subset \operatorname{int} Q$. (See S3.)

By its very definition this quadrangle has the property that, for any $\nu_{0, p}^{*} \in \operatorname{int} Q$ we have that

$$
\beta_{i k}=\measuredangle \nu_{0, p} A_{i} A_{k}>\frac{\alpha_{i}}{4} \geq \frac{\phi_{0}}{4} ; k \in\{(i-1) \bmod 4,(i+1) \bmod 4\} .
$$

Also,

$$
\gamma_{i k}^{*}=\measuredangle A_{i} \nu_{0}^{*} A_{k}>\min \left\{\measuredangle A_{i-1} A_{i} A_{i+1}, \measuredangle A_{i} A_{i+2} A_{i-1}\right\} .
$$

(See Figure 5.) But one of the angles $\measuredangle A_{i-1} A_{i} A_{i+1}$ and $\measuredangle A_{i} A_{i+2} A_{i-1}$ belongs to one of the original fat triangles, so

$$
\gamma_{i k}^{*}>\phi_{0} .
$$

So, from (3.3) and (3.5) it follows that the triangles $\triangle A_{i} \nu_{0}^{*} A_{i+1} ; i=$ $0, \ldots, 3(\bmod 4)$ are fat.

(b) In this case, rather than tracing our steps back through the same argument as in the previous case, we prefer to dissect $Q$ into two triangles and one convex quadrilateral, in the following way: If $\square A_{0} A_{0} A_{0} A_{3}$ is such that $\measuredangle A_{3} A_{0} A_{1}>\pi$ and such that $\nu_{0, p} \in A_{2}$, then consider the bisectors $A_{0} B_{12}$ of $\measuredangle A_{1} A_{0} A_{2}$ and $A_{0} B_{23}$ of $\measuredangle A_{2} A_{0} A_{3}$. 


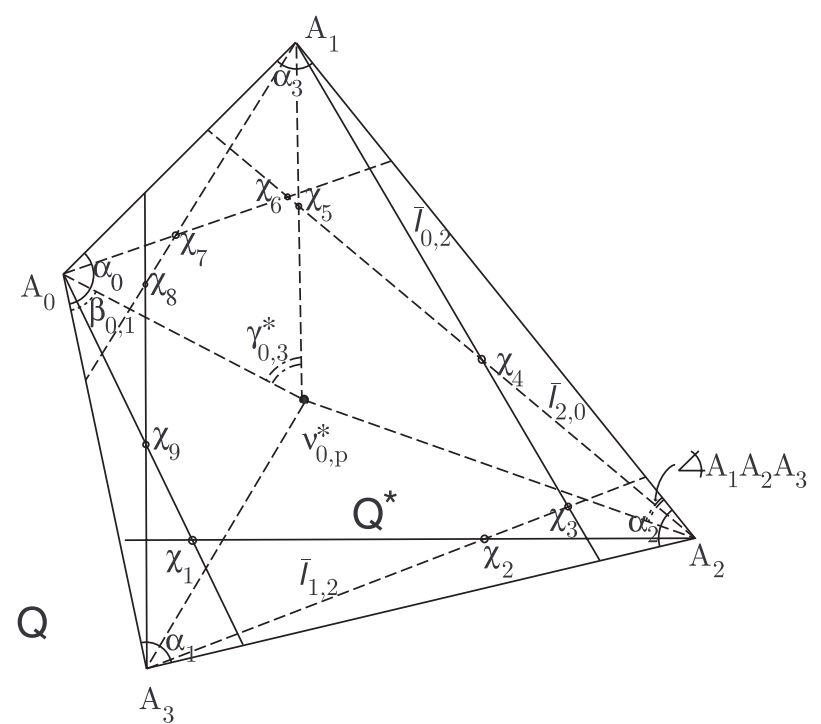

FigURE 4.

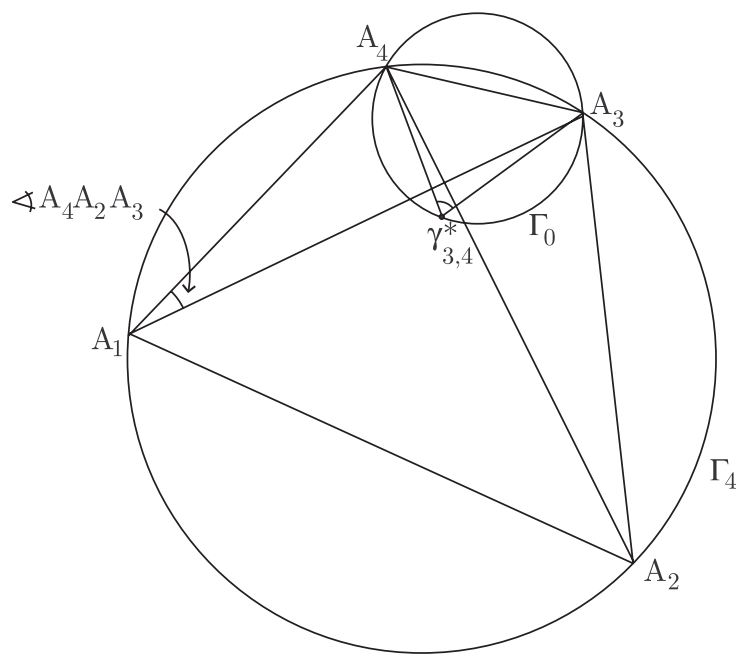

FiguRE 5.

Now, instead of returning once again to the argument used in case (a), it is easy to proceed directly and show that the triangles $\triangle A_{1} A_{0} B_{12}, \ldots, \triangle A_{0} B_{23} A_{3}$ are fat. (See [S3] for details.)

We are now faced with a new fat triangulation. However, new points of intersection with $e_{0, l}$ are introduced: Let them be $\nu_{0, p-1}$ and $\nu_{0, p+1}$. If

$$
e_{0, l} \cap \square A_{0} B_{12} A_{2} B_{23} \cap\left\{B_{12} B_{23}\right\}=\emptyset,
$$


then the same argument we used for the original triangulation shows that $\nu_{0, p-1}$ and $\nu_{0, p+1}$ are $\geq \phi_{0}$. If

$$
e_{0, l} \cap \square A_{0} B_{12} A_{2} B_{23} \cap\left\{B_{12} B_{23}\right\} \neq \emptyset,
$$

we can employ either one of the following two methods to remedy the situation: (a) use the general position technique again and bring the new triangulation to the required position; or (b) consider, instead of the bisector $A_{0} B_{23}$, the meridian $A_{0} M_{23}\left(M_{23} \in A_{2} A_{3}\right)$ - see [S3] for the details.

We still have to face the problem of mashing the triangulations 'over the wave front' of the $s_{i}$-s. Away from the vertices of the complex $\mathcal{S}$, we are faced with two possibilities: $\mathcal{S}$ contain:

(a) one, or

(b) two of the vertices of $\sigma \in \Sigma$.

We shall deal first with Case (b).

Case (b). By further dividing the triangles of

$$
\text { Front } \mathcal{S}=\{s \in \mathcal{S} \mid \exists e-\text { edge of } s \text { such that } e \in \partial \mathcal{S}\},
$$

we are able to 'erase' the families $\mathcal{S}_{1}$ and $\mathcal{S}_{2}$, where $\mathcal{S}_{1} \cup \mathcal{S}_{2}=$ Front $\mathcal{S} \cap\left\{e_{i}\right\}$, $i=1,2$ (see Figure 6(a),(c)). Since we are in the planar case, the division may be done using lines parallel to the edges of $s$. Still, some of the simplices $s \in \mathcal{S}$ may intersect the edges $e_{i}$ of $\sigma$ at an angle $\phi_{s, l_{i}}<\phi_{0}, i=1,2$. However, the angles $\phi_{s, l_{i}}^{2}$ (see Figure 5(b)) are, by the previous argument, at least $\geq \phi_{0}$.

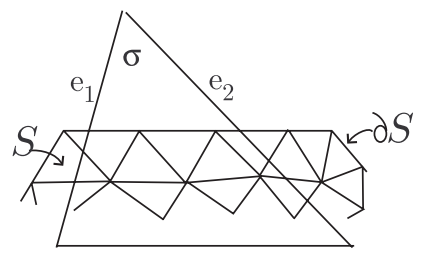

(a)

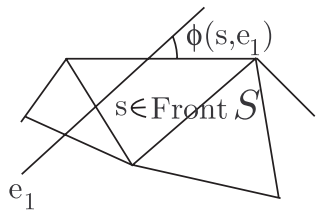

(b)

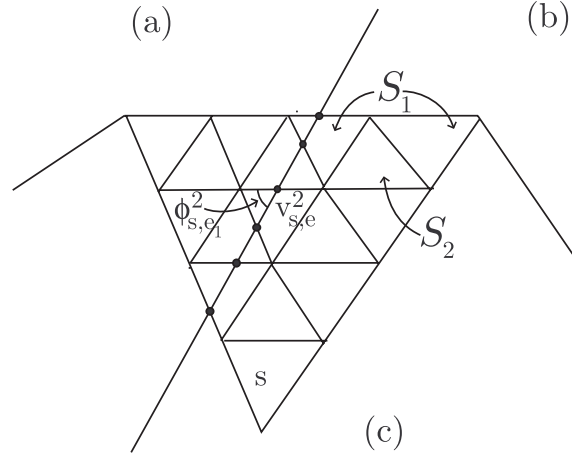

FigURE 6.

Let $\mathcal{S}^{\prime}=\mathcal{S} \backslash$ Front $\mathcal{S}$. Then $\partial \mathcal{S}^{\prime}$ will still be convex, so we can consider the joins (cones) $J\left(v_{12}, \varepsilon_{k}\right)$, where $\varepsilon_{k}$ are the edges of $\partial \mathcal{S}^{\prime}$ that are included in $\sigma$, and $v_{12}=e_{1} \cap e_{2}$ (see Figure 7).

Note that the inradii $r_{0}$ and circumradii $R_{0}$ of the simplices $\sigma_{0} \subset N_{e}^{*} \backslash N_{e}^{* \prime}$, are uniformly bounded (see [Pe]). 


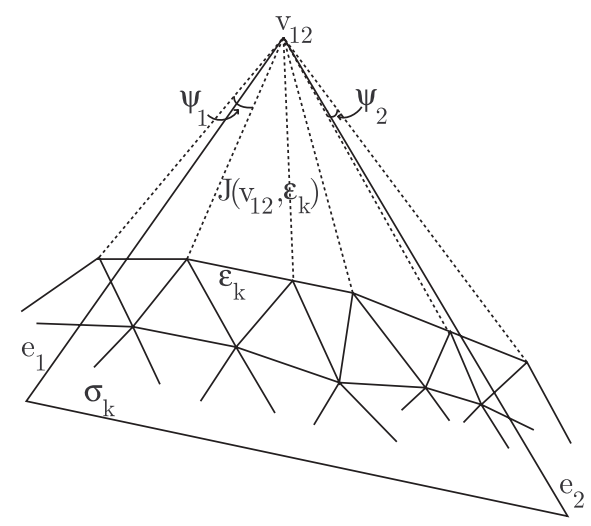

FiguRE 7.

Thus, there exist numbers $m_{0}$ and $m_{1}$ such that

$$
m_{0} \leq \operatorname{diam}(\sigma) \leq m_{1} \text {; for any } \sigma \text { such that } \bar{\sigma} \cap T_{0} \neq \emptyset .
$$

Therefore, there exists $k_{1} \in N_{+}$such that

$$
\frac{1}{10^{k_{1}}} \operatorname{diam}(\sigma) \leq \operatorname{diam}(s) ; \text { for any } s, \sigma \text { such that } \bar{s}, \bar{\sigma} \cap T_{0} \neq \emptyset \text {. }
$$

This, in conjunction with (3.2), assures us that the number $\rho_{1}$ of triangles $s_{i}$ that intersect the edge $e_{0, l}$ (see Figure $8(\mathrm{a})$ ) will be bounded by two natural numbers, i.e. there exist $n^{1}, n^{2} \in \mathbb{N}$ such that

$$
n^{1} \leq \rho_{1} \leq n^{2} .
$$

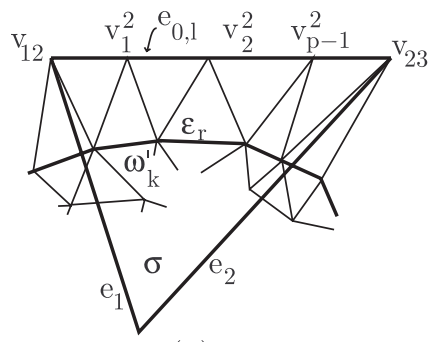

(a)

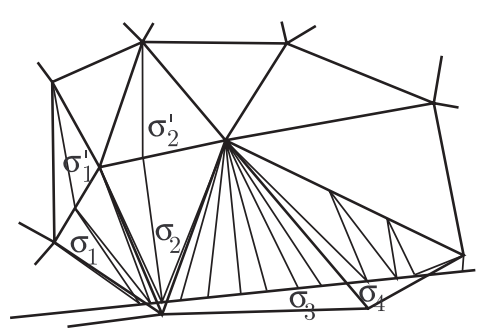

(b)

FiguRe 8.

Moreover, our last subdivision ensures us that the number $\rho_{2}$ of triangles intersecting $\partial \mathcal{S}^{\prime}$ is

$$
\rho_{2} \leq 3\left(\rho_{1}-1\right)+2,
$$

so there exists $\lambda_{0}>0$ such that the angles $\psi_{k}=\measuredangle v_{12}, \psi^{1}, \psi^{2}$ (see Figure 7) satisfy

$$
\psi_{k} \geq \lambda_{0} \varphi_{0}, \psi^{i} \geq \lambda_{0} \varphi_{0}, i=1,2 ;
$$

as desired.

We still have to deal with Case (a). 
Case (a). We repeat the procedure used for the subdivision of $\partial \mathcal{S}$ and Front $\mathcal{S}$ that we employed in Case (b). We divide the edge $e_{2}$ into $\rho_{2}-1$ equal segments and consider the joins (cones) $J\left(v_{j}^{2}, \varepsilon_{j+1}\right)$ and $J\left(w_{k}^{\prime}, v_{k}^{2} v_{k+1}^{2}\right)$ (see Figure $8(\mathrm{a})$ ). Using the same arguments as before, one easily checks that the resulting triangulation of $s \backslash \mathcal{S}$ will be fat. Moreover, although this procedure dramatically reduces the fatness of the next stratum of simplices of the family $\Sigma$, it leaves the other strata unchanged.

This procedure takes care of the intersections of $\Sigma$ with 'the front wave' $N_{e}^{*}$. To deal with the case $N_{e}^{* \prime}$ one proceeds along the same lines and then fits the new triangulation $\mathcal{S}^{\prime}$ to $\mathcal{S}$ in a properly chosen tubular region $J_{1}$, using, instead of $\sigma$, triangles $s_{0} \in \mathcal{S} \cap \mathcal{T}_{1}$.

We have yet to contend with the problem posed by 'corners', i.e. by triangles $s \in \mathcal{S}$ such that (i) $s \in$ Front $\mathcal{S}$ and (ii) $\partial s \cap \partial \mathcal{S}=v$ (where $v$ is a vertex). The two cases that ensue may be treated with the methods developed before. (See [S3].)

This concludes the fattening process for the two-dimensional triangulations.

3.3. Fattening 3-dimensional triangulations. We shall divide the fattening process of two intersecting 3-dimensional tetrahedra into two parts: (A) the fattening of the 2-dimensional intersection between the triangles belonging to $\partial \mathcal{S}$ and a face $f_{123}=\triangle v_{1} v_{2} v_{3}$ of a tetrahedron $\sigma \in \Sigma$, and (B) the extension of the new triangulation to a fat 3 -dimensional triangulation.

3.3.1. Fattening 2-dimensional intersections. Let us start by noticing that, since the fatness of the tetrahedra $s_{i} \in \mathcal{S}$ is bounded from below, it follows from the trihedral sinus formula (see [Ber, 18.6) that their dihedral angles will also be bounded from below. Therefore, even after the partition of $f_{123}$ into triangles $\left\{\tau_{k}\right\}$ and quadrilaterals $\left\{\eta_{j}\right\}$, into a triangulation $\left\{\tilde{\tau}_{k}\right\}$, the number of triangles around each vertex will be bounded by a natural number $m_{1}$. We shall exploit this fact to our advantage, so that we will be able to replace $\left\{\tilde{\tau}_{k}\right\}$ by a fat triangulation $\left\{\tilde{\tau}_{k}^{*}\right\}$ that is 'close' to $\left\{\tilde{\tau}_{k}\right\}$.

Indeed, if we denote by $\tilde{\alpha}_{j}^{0}, j=1, \ldots, \bar{m}_{0}, \bar{m}_{0} \leq m$; the angles of the triangles $\left\{\tilde{\tau}_{k}^{0}\right\}$ that are respectively adjacent to the vertex $\tilde{\nu}^{0}=\bigcap_{j} \tilde{\tau}_{j}^{\prime}$ (see Figure 9), then there are two sources of 'thinness': either $\tilde{\alpha}_{j}^{0}=\alpha_{j}^{0}$ is smaller than $\varphi_{0}$, for some $j_{0} \in\{1, \ldots, \bar{m}\}$ or one of the angles $\tilde{\alpha}_{j_{1}}^{0} \tilde{\alpha}_{j_{1}+1}^{0}$ produced by the division of the quadrilateral $\eta_{1}$ into two triangles, $\tilde{\tau}_{j_{1}}^{0}$ and $\tilde{\tau}_{j_{1}+1}^{0}$.

If we bisect the angles $\tilde{\alpha}_{j}^{0}, j=1, \ldots, \bar{m}_{0}$, we will receive angles $\tilde{\alpha}_{j k}^{0}, j=$ $1, \ldots, \bar{m}_{0} ; k=1,2$. Let us consider the angles $\tilde{\beta}_{j k}^{0}, j=1, \ldots, \bar{m}_{0} ; k=1,2$, where $\tilde{\beta}_{j 1}^{0}=\frac{\tilde{\alpha}_{j-1}^{0}+\tilde{\alpha}_{j}^{0}}{2}, \tilde{\beta}_{j 2}^{0}=\frac{\tilde{\alpha}_{j}^{0}+\tilde{\alpha}_{j+1}^{0}}{2}$. If each and every of the angles $\tilde{\beta}_{j k}^{0}$ defined above is greater than $\varphi_{1}=\varphi_{0} / 10$, then we desist and proceed towards part (B). But it may be that, for instance, both $\tilde{\alpha}_{j-1}^{0}$ and $\tilde{\alpha}_{j}^{0}$ are smaller than $\varphi_{0} / 5$. If this happens, we continue the process of 'mixing the angles'. To be more precise, let us delate - for conciseness - the upper index ' 0 ' in the enumeration of the angles ' $\beta$ ' and denote them by $\tilde{\beta}_{j}, j=1, \ldots, \bar{m}_{0}$ and let us form the sequence of angles $\tilde{\beta}_{j}^{\prime}, \tilde{\beta}_{j}^{\prime \prime}, \tilde{\beta}_{j}^{\prime \prime \prime}$, etc. , where $\tilde{\beta}_{j}^{\prime}=\frac{1}{2}\left(\tilde{\beta}_{j-1}+\tilde{\beta}_{j+1}\right), \tilde{\beta}_{j}^{\prime \prime}=\frac{1}{2}\left(\tilde{\beta}_{j-1}^{\prime}+\tilde{\beta}_{j}^{\prime}\right)$, and so on. But this process will halt-inasmuch as we are concerned - in a finite number of steps, for the following inequality holds:

$$
\tilde{\beta}_{j}^{\bar{m}_{0}}>\frac{\alpha_{1}+\cdots+\alpha_{\bar{m}_{0}}}{2^{\bar{m}_{0}+1}} ; j=1, \ldots, \bar{m}_{0}
$$




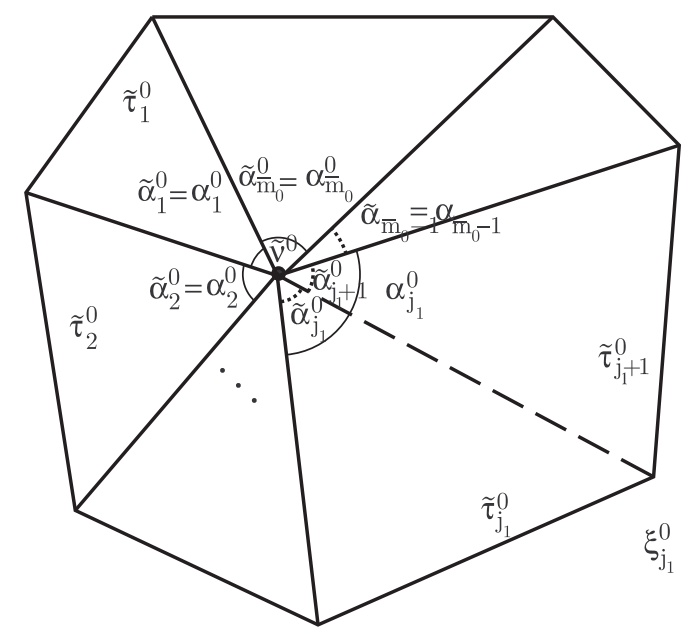

FiguRE 9.

That is,

$$
\beta_{j}^{\bar{m}_{0}+1}>\frac{2 \pi}{2^{\bar{m}_{0}+1}}>\frac{\varphi_{0}}{10^{k}} ; j=1, \ldots, \bar{m}_{0}
$$

hence,

$$
\beta_{j}^{\bar{m}_{0}-1}>\varphi_{1}=\frac{\varphi_{0}}{10^{\bar{k}}} ; 1, \ldots, \bar{m}_{0} ;
$$

where $\bar{k}$ is the least natural power that satisfies the right-handed inequality in (4.12).

We shall use the bound above in order to produce a fat triangulation. However, some care is needed in doing this, for in general, both the number of iterations used for each vertex and the number of bisectors $b_{j}^{k}, j=1, \ldots, \bar{m}_{0} ; k=1,2,3$; that intersect the triangle $\tilde{\tau}$ will be different, thus affecting the sizes of the angles $\tilde{\alpha}_{j}^{k}$.
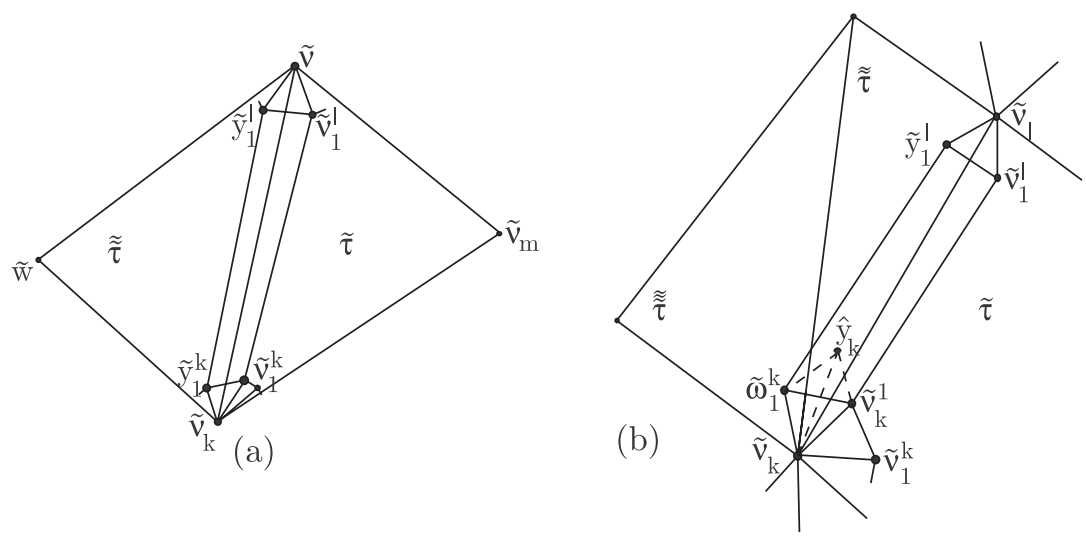

FiguRE 10. 
Let $b$ denote the shortest of the segments $b_{j}^{k} \cap \tilde{\tau}, k=1,2,3$. (By elementary geometry, $b$ should be the segment $b_{i_{0}}^{k_{0}}$ that is the nearest to the shortest side of $\tilde{\tau}$, where $\tilde{\alpha}^{k_{0}}$ is the smallest angle of $\tilde{\tau}$.) Moreover, let us consider segments $\tilde{\nu}_{k} \nu_{i}^{k} \subset b_{i}^{k}$, such that $\tilde{\nu}_{k} \nu_{i}^{k}=b / 10^{k_{2}}=\hat{b}$ where $k_{2}$ is chosen in such a manner that the quadrilateral $\square \nu_{1}^{k} \nu_{1}^{l} y_{1}^{k} y_{1}^{l}$ is simple; where $\nu_{1}^{k}, \nu_{1}^{l}$ denote the vertices closest to the edge $\nu_{k} \nu_{l}$ and $y_{1}^{k}, y_{1}^{l}$ play the same role in the adjacent triangle $\tilde{\tilde{\tau}}$ (see Figure 10(a)). It may be that we will have to consider $\square \nu_{1}^{k} \nu_{1}^{l} \tilde{y}_{1}^{k} \tilde{y}_{1}^{l}$ (or any permutation of indices)-see Figure 10(b). (Here $\tilde{w}_{1}^{k}$ plays in the triangle $\tilde{\tilde{\tau}}$; the role $\tilde{\nu}_{1}^{k}$ plays in the triangle $\tilde{\tau}$.) In this case consider a point $\hat{y}_{k} \in$ int $\tilde{\tilde{\tau}}$, such that:

(i) $\hat{y}_{k} \hat{\nu}_{k}=\hat{b}$

(ii $\left.i_{1}\right) \measuredangle \tilde{w}_{1}^{k} \hat{\nu}_{k} \hat{y}_{k} \geq \frac{\varphi_{1}}{2}=\varphi_{2}$;

(ii $\left.i_{2}\right) \measuredangle \hat{y}_{k} \hat{\nu}_{k} \hat{\nu}_{1}^{k} \geq \frac{\varphi_{1}}{2}=\varphi_{2}$.

Remark 3.2. The existence of the positive integer $k_{2}$ with the desired properties is guaranteed by the fact that the angles $\tilde{\alpha}^{k}, \tilde{\alpha}^{l}, \tilde{\alpha}_{i}^{k}, \tilde{\alpha}_{i}^{l}, \tilde{\alpha}^{m}$ and $\tilde{\tilde{\alpha}}^{m}$ are $\geq \varphi_{1}$, thus

$$
\begin{aligned}
c_{1} b & \leq \tilde{\nu}_{k} \tilde{\nu}_{k} \leq c_{2} b ; \\
c_{1}^{\prime} b^{\prime} & \leq \tilde{\nu}_{k} \tilde{\nu}_{k} \leq c_{2}^{\prime} b^{\prime} .
\end{aligned}
$$

In consequence we are facing the situation depicted in Figure 11, where we also illustrated the conning of the segments $\tilde{\nu}_{i}^{k} \tilde{\nu}_{i+1}^{k}$ and $\tilde{\nu}_{1}^{k} \tilde{\nu}_{1}^{l}$ from the barycenter $\tilde{\tilde{b}}$ of $\tau ; k=1,2,3 ; l=1,2,3$.

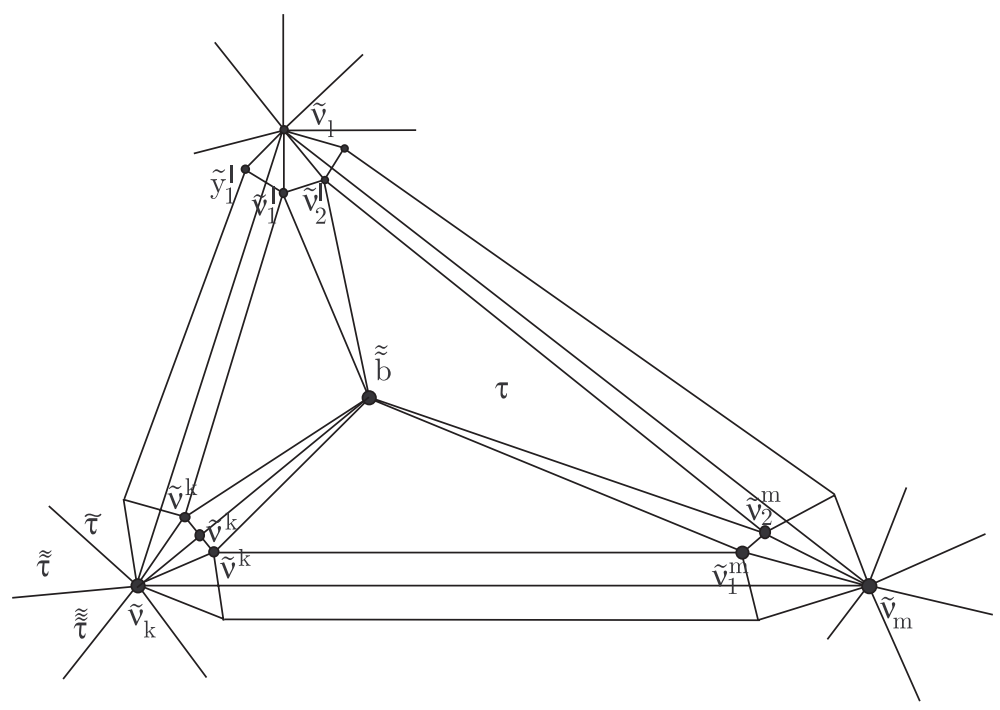

Figure 11.

Now we have to show the fatness of three types of polygons:

(1) triangles $T_{i j}=\triangle \tilde{\nu}_{i}^{k} \tilde{\nu}_{j}^{l} \tilde{\tilde{b}}$;

(2) quadrilaterals $Q_{k l}=\square \tilde{\nu}_{1}^{k} \tilde{\nu}_{1}^{l} \tilde{y}_{1}^{l} \tilde{y}_{1}^{k}$;

(3) triangles $T^{k l}=\triangle \tilde{\nu}_{1}^{k} \tilde{\nu}_{1}^{l} \tilde{\tilde{b}}, k, l=1,2,3$. 
We start by noticing that fatness of a quadrilateral means that:

(a) its angles are bounded from below, and;

(b) the ratios $l_{\lambda} l_{\iota}, \lambda, \iota=1, \ldots, 4 m$ between the lengths of its sides are also bounded from below.

An easy computation (see [S3] ) shows that

$$
\begin{gathered}
\tilde{y}_{1}^{l} \tilde{\nu}_{1}^{l} \geq 2 \hat{b} \sin \frac{\pi}{\bar{m}_{0}} ; \\
\tilde{y}_{1}^{l} \tilde{\nu}_{1}^{k} \geq \tilde{\nu}_{1}^{k} \tilde{\nu}_{1}^{l}-2 \hat{b} ; \\
\measuredangle \tilde{\nu}_{1}^{l} \geq \arctan \frac{\hat{b} \cos \frac{2 \pi}{\hat{m}_{0}}}{\tilde{\nu}^{k} \tilde{\nu}^{l}-2 \hat{b} \sin \frac{2 \pi}{\bar{m}_{0}}} ;
\end{gathered}
$$

where $\hat{b}=\tilde{\nu}^{k} \tilde{y}_{1}^{k}$. Similar formulas hold for the other pairs of vertices.

Therefore, the arguments employed before show that $\square Q_{k l}$ is decomposable into fat triangles, so case (2) is dealt with.

In a manner similar to that used in case (2), we show that the triangles of types $T_{i j}$ and $T_{k l}$ are also fat; indeed:

$$
\measuredangle \tilde{\nu}_{i} \tilde{\tilde{b}} \tilde{\nu}_{i+1}^{k}<2 \arctan \frac{3 \pi}{2 \bar{m}_{0} b^{l}}
$$

where

$$
\tilde{y}_{1}^{l} \tilde{\nu}_{1}^{k} \geq b^{l} \geq \tilde{y}_{1}^{l} \tilde{\nu}_{1}^{m}
$$

and also

$$
\measuredangle \tilde{\nu}_{1}^{l} \tilde{\tilde{b}} \tilde{\nu}_{1}^{k}>\measuredangle \tilde{\nu} l \tilde{\tilde{b}} \tilde{\nu}^{k}>\measuredangle \tilde{\nu}^{l} \tilde{\nu}^{m} \tilde{\nu}^{k} \geq \varphi_{1}
$$

and so we dispose with cases (1) and (3) too, thus concluding the proof of part (A).

3.3.2. The extension to a fat 3-dimensional triangulation. We start by observing that, if $u$ is a vertex of the simplex $s_{i}$ such that $f_{123} \cap s_{i}=\tilde{\tau}$-where $f_{123}$ is a face of the tetrahedron $\sigma$-then the triangles $T^{k l}, T_{i j}$ and those produced by the subdivision of $Q_{k l}$ may also be coned from $u$.

We want to show that the simplices thus generated - denoted by $V_{i j}^{\delta}=J\left(u_{\delta}, T_{i j}\right)$, $\delta=1,2,3,4$; etc. - have big angles. We shall justify this affirmation for tetrahedra of type $V_{i j}^{\delta}$, the other cases being completely analogous. Indeed:

(a) The angles $\tilde{\nu}_{i}^{k}, \tilde{\nu}_{j}^{k}$ and $\measuredangle \tilde{\tilde{b}}$ are big by the very construction of $\triangle \tilde{\nu}_{i}^{k} \tilde{\nu}_{j}^{k} \tilde{\tilde{b}}$.

(b) The plane angles around the vertex $u_{\delta}$ are big, since $\tilde{\nu}_{i}^{k} \tilde{\nu}_{j}^{k} \geq c_{1} b$, and because $\tilde{\nu}_{i}^{k} \tilde{\nu}_{j}^{k}$ is included in the plane of $\tilde{\tau}$.

(c) The dihedral angles around $u_{\delta}$ are also big (by the argument above and by the tetrahedral sinus formula). However, the basic type of small angles may still occur (see Figure 12, where they are denoted by ' $s$ ').

In fact, the case of Figure 12(a) is associated with a low height/base side ratio; whereas, in this instance it follows (by the fatness of the tetrahedron $u_{1} u_{2} u_{3} u_{4}$ ) that

$$
\frac{u_{\delta} \tilde{O}_{i j}}{\tilde{\nu}_{i}^{k} \tilde{\nu}_{j}^{k}}>\frac{u_{\delta} \tilde{O}_{i j}}{\tilde{\nu}_{l} \tilde{\nu}_{m}}>c^{0}
$$

where $c^{0}$ is a constant, so this case is excluded. 


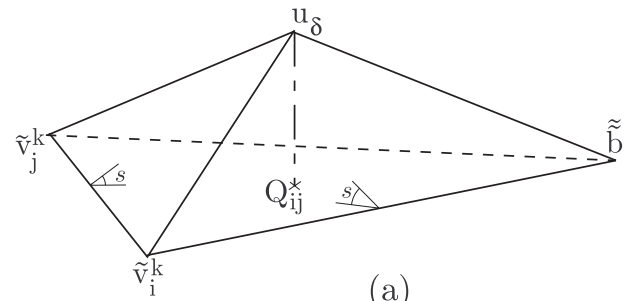

(a)

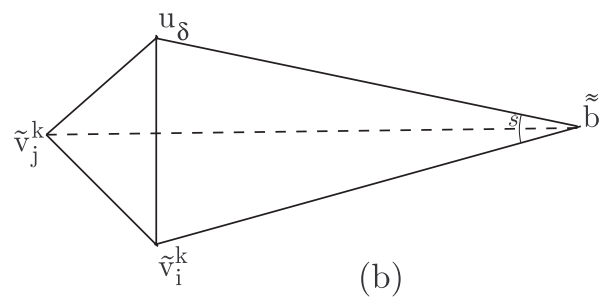

(b)

FiguRE 12.

Part of the angles covered by case (b) of Figure 12 are big, for the following inequality (and its analogues hold)

$$
\frac{u_{\delta} \tilde{\nu}_{i}^{k}}{\tilde{\nu}_{i}^{k} \tilde{\nu}_{j}^{k}}>c^{*} \frac{u_{\delta} \tilde{\nu}_{k}}{\tilde{\nu}_{l} \tilde{\nu}_{k}}
$$

where $c^{*}$ is a constant.

For a full proof of the fatness of $u_{\delta} \tilde{\nu}_{i}^{k} \tilde{\nu}_{j}^{k} \tilde{\nu}_{k}$ (to wit) we still have to check the size of each of the following angles: $\measuredangle \tilde{\nu}_{i}^{k} \tilde{\nu}_{j}^{k}, \measuredangle \tilde{\nu}_{k} u_{\delta} \tilde{\nu}_{i}^{k}$ and $\measuredangle \tilde{\nu}_{k} u_{\delta} \tilde{\nu}_{j}^{k}$, where $\measuredangle \tilde{\nu}_{i}^{k} \tilde{\nu}_{j}^{k}$ denotes the dihedral angle between the faces $\triangle u_{\delta} \tilde{\nu}_{i}^{k} \tilde{\nu}_{j}^{k}$ and $\triangle \tilde{\nu}_{k} \tilde{\nu}_{i}^{k} \tilde{\nu}_{j}^{k}$.

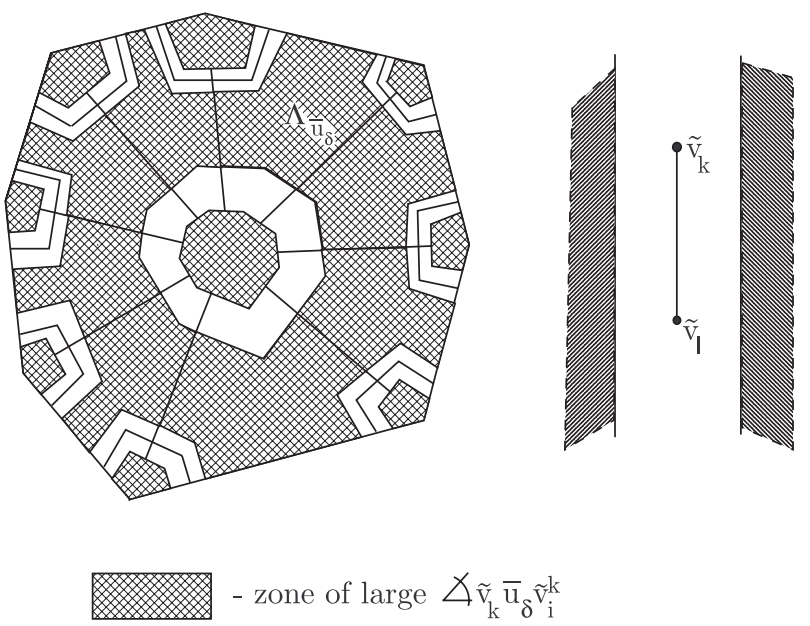

Figure 13.

To ensure the proper size of the last two angles one has to notice that the size of - e.g. $\measuredangle \tilde{\nu}_{k} u_{\delta} \tilde{\nu}_{i}^{k}$-is inversely proportional to:

(i) the distance $\hat{\delta}$ from $u_{\delta}$ to $\tilde{\nu}_{l}^{k} \tilde{\nu}^{k}$, and

(ii) the distance $\delta^{*}$ from $\bar{u}_{\delta}$ to $\tilde{\nu}_{l}^{k} \tilde{\nu}^{k}$. (Here $u_{\delta} \bar{u}_{\delta} \perp f_{123}$.)

But, since the lengths $\tilde{\nu}_{l}^{k} \tilde{\nu}^{k}$ are bounded from below, there exists a minimal universal distance $\delta_{0}^{*}$ such that if $\delta^{*}<\delta_{0}^{*}$, then $\measuredangle \tilde{\nu}_{k} u_{\delta} \tilde{\nu}_{j}^{k}>\varphi_{2}^{*}$, for a suitable $\varphi_{2}^{*}$.

By eventually decreasing $\delta_{0}^{*}$ to a new $\delta_{1}^{*}$, we can ensure that the angles of type $\measuredangle \tilde{\nu}_{k} u_{\delta} \tilde{\nu}_{i}^{k}$ are strictly greater than some $\varphi_{2}^{* *}, \varphi_{2}^{* *} \leq \varphi_{2}^{* *}$, for each $\tilde{\nu}_{k} \in S t\left(u_{\delta}\right) \cap f_{123}$ and for each $\tilde{\nu}_{i}^{k}$, which concludes the proof of case (b). Indeed, we can vary the 
position of $\bar{u}_{\delta}$ in the region $\Lambda_{\bar{u}_{\delta}}$, such that all the required angles will be large- see Figure 13. (Such a small movement won't affect the fatness of the next stratum of tetrahedra of type $s$.)

After disposing with this case, we can turn our attention to case (a). Now, since $\left|\tan \measuredangle \tilde{\nu}_{i}^{k} \tilde{\nu}_{j}^{k}\right|=\frac{u_{\delta} \bar{u}_{\delta}}{\bar{u}_{\delta} \bar{u}_{\delta}}$, where $u_{\delta} \bar{u}_{\delta} \perp\left(\tilde{\nu}_{i}^{k} \nu_{k} \tilde{\nu}_{j}^{k}\right), \bar{u}_{\delta} \overline{\bar{u}}_{\delta} \perp \tilde{\nu}_{i}^{k} \tilde{\nu}_{j}^{k}$, and since $\bar{u}_{\delta} \overline{\bar{u}}$ is known, we only have to ensure that $u_{\delta} \bar{u}_{\delta}$ is bounded from below.

For this, one essentially makes use of the very specific form of the triangulation of the geometric neighbourhood of an elliptic axis.

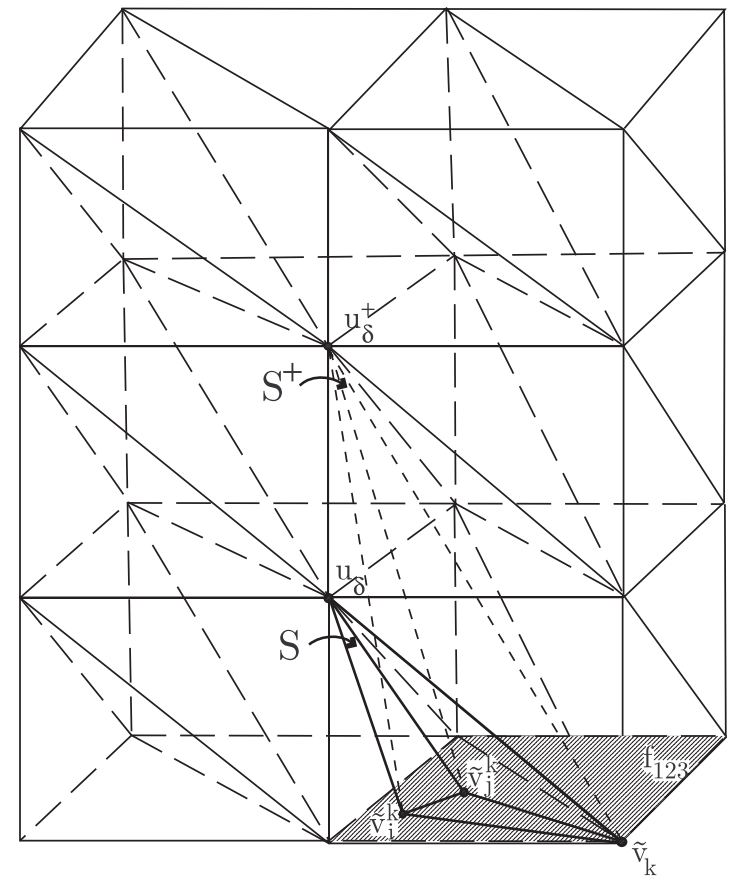

FiguRe 14.

Namely, instead of considering tetrahedra $u_{\delta} \tilde{\nu}_{i}^{k} \tilde{\nu}_{j}^{k} \tilde{\nu}_{k}$, we shall consider tetrahedra $u_{\delta}^{+} \tilde{\nu}_{i}^{k} \tilde{\nu}_{j}^{k} \tilde{\nu}_{k}$ where $u_{\delta}^{+}$is the vertex corresponding to $u_{\delta}$ in the next stratum of ' $s$ '-type tetrahedra, of the family $\mathcal{S}$. (See Figure 14.)

Now the desired bound - for $\frac{u_{\delta}^{+} \bar{u}_{\delta}^{+}}{\bar{u}_{\delta}^{+} \bar{u}_{\delta}^{+}}$instead of $\frac{u_{\delta} \bar{u}_{\delta}}{\bar{u}_{\delta} \bar{u}_{\delta}}$-is achieved, whilst the very existence of the constants obtained before is not changed, only their magnitude; we shall denote them by an upper-right superscript: e.g. $\varphi_{2}^{++}$.

Remark 3.3. The method we have just used is adaptable to the general context: consider-instead of $u_{\delta}$-vertices $u_{\delta}^{++}$, such that dist $t_{\text {eucl }}\left(u_{\delta}, u_{\delta}^{++}\right)=h_{0}$, where $h_{0}=\frac{1}{3} h_{\min }$, and $h_{\min }=$ the minimal height of the simplices $s^{+} \in S t\left(u_{\delta}\right)$. The points $u_{\delta}^{++}$are to be chosen on the normal through $u_{\delta}$ to the plane $f_{123}$, so that the combinatorics of the triangulation will suffer no alteration. Clearly, uniform bounds will again be attained.

In order to conclude the proof of Theorem 1.1 we still have to provide for a fat triangulation around the node points. However, this missing case is easily dealt with 
by considering the intersections of the geometric neighbourhoods of the elliptic axes. Such an intersection will automatically inherit from the tubular neigbourhoods that generate it a natural, stratified, fat triangulation. (See Section 3.) So, the arguments involved in the proof of the restricted case of axes that do not intersect do apply here, too. Thus we conclude the proof of Theorem 1.1.

\section{The EXISTEnCE of QUASIMEROMORPHIC MAPPINGS}

The technical ingredient in Alexander's trick is the following lemma:

Lemma 4.1 ([MS1], $\mathrm{Pe}$ ). Let $M^{3} \subset \mathbb{R}^{N}$ be an embedded orientable 3-manifold, let $\mathcal{T}$ be a chessboard fat triangulation of $M^{3}$, let $\sigma \in \mathcal{T}, \sigma=\left(p_{0}, \ldots, p_{n}\right)$ and let $\tau_{0}=\left(p_{0,1}, \ldots, p_{0,3}\right)$ denote the equilateral 3 -simplex inscribed in the unit sphere $\mathbb{S}^{2}$. Then there exists an orientation-preserving homeomorphism $h=h_{\sigma}:|\sigma| \rightarrow \widehat{\mathbb{R}^{3}}$ s.t.

(1) $h(|\sigma|)=\left|\tau_{0}\right|$, if $\sigma$ is positively oriented and $h(|\sigma|)=\widehat{\mathbb{R}^{3}} \backslash\left|\tau_{0}\right|$, otherwise.

(2) $h\left(p_{i}\right)=p_{0, i}, i=0, \ldots, 3$.

(3) $\left.h\right|_{\partial|\sigma|}$ is a PL-homeomorphism.

(4) $\left.h\right|_{\text {int }|\sigma|}$ is quasiconformal.

Proof. If $\operatorname{det}\left(p_{0}, \ldots, p_{3}\right)>0$, then the $P L$-mapping $h$ defined by condition (2) above also satisfies conditions (1), (3), and (4). If $\operatorname{det}\left(p_{0}, \ldots, p_{3}\right)<0$, we define $h$ as follows: $h=\varphi^{-1} \circ J \circ \varphi \circ h_{0}$, where $\varphi$ is the radial linear stretching $\varphi: \tau_{0} \rightarrow \mathbb{R}^{3}$, $J$ denotes the reflection in the unit sphere $\mathbb{S}^{2}$ and $h_{0}:|\sigma| \rightarrow\left|\tau_{0}\right|$ is the orientationreversing $P L$-mapping defined by condition (2). Recall that $\varphi$ is onto and bilipschitz (see [MS2]). Moreover, by a result of Gehring and Väisalä, $\varphi$ is also quasicomformal (see $[\mathrm{V}]$ ). We can extend $\varphi$ to $\widehat{\mathbb{R}^{3}}$ by defining $\varphi(\infty)=\infty$. It follows that $h$ indeed represents the required $P L$-homeomorphism.

Remark 4.2. The existence of the integer ' $N$ ' in the statement of Lemma 4.1 above is guaranteed by Nash's Theorem (see, e.g. $[\mathrm{Pe}$, $[\mathrm{Spi} \mathrm{V}]$ ).

The Existence Theorem of quasimeromorphic mappings immediately follows.

Proof of Theorem 1.6. Let $\mathcal{T}$ be the $\varphi_{0}^{*}$-fat chessboard triangulation of $\mathbb{H}^{3} / G$ constructed in Section 3 above. Let $f: \mathbb{H}^{3} / G \rightarrow \widehat{\mathbb{R}^{3}}$ be defined by $\left.f\right|_{|\sigma|}=h_{\sigma}$, where $h$ is the homeomorphism constructed in the lemma above. Then $f$ is a local homeomorphism on the 2-skeleton of $\widetilde{\mathcal{T}}$ too, while its branching set $B_{f}$ is the 1 -skeleton

of $\tilde{\mathcal{T}}$. By its construction, $f$ is quasiregular and its (outer) dilatation depends only on $\varphi_{0}^{*}$ (and on the dimension $n=3$ ) - see $\left[\mathrm{Tu}\right.$, Lemma E. The lift $\tilde{f}$ of $f$ to $\mathbb{H}^{3}$ represents the required $G$-automorphic quasimeromorphic mapping.

\section{Higher Dimensions}

Our approach to the extension of our results to dimension $n \geq 4$ - which we will discuss only briefly - is based upon reduction to smaller dimensions. To do this, let us observe that if $S(n+1)$ is a simplex in $R^{n}$, then any sectioning hyperplane separates the vertices of $S(n+1)$ into two groups of $p$ and $n+1-p$ vertices, respectively. The notation is as follows: $(p, n+1-p) \equiv(n+1-p, p)$ for the polytopes of the section, and $(p \mid n+1-p)$ and $(n+1-p \mid p)$ for the resulting frusta. (See Som for precise definitions.)

The reduction to lower dimensions is permitted by the following. 
Lemma 5.1 ( $\underline{\text { Som }})$. The frustum of type $(p \mid 2)$ of $S(p+q)$ is isomorphic to the section of type $(p, q+1)$ of $S(p+q+1)$.

The fattening algorithm is, in a nutshell, as follows:

(i) Divide the polytopes obtained by sectioning into simplices: first the section polytope, then the faces that are part of the original $S(n+1)$, while ensuring fatness by the methods discussed in Section 3. If needed, apply an inductive process on the dimension of the simplices.

(ii) Fatten the frusta by proving the existence of a locus of points where from all the $(n-k)$-faces are seen at big $(n-k)$-dimensional angles. (See Som for the relation between the $p$-dimensional angles of an $n$-simplex, $p=2, \ldots, n-1$.) The existence of the said locus follows from [CMS, Lemma 6.3.

The proof of Theorem 1.1 now follows along the same lines as in the 3 -dimensional case. However, to produce the desired geometric neighbourhoods, we have to more clearly understand the geometry of the elliptic locus of a Kleinian group with torsion, acting on $\mathbb{H}^{n}, n \geq 4$. We are, nevertheless, fortunate, for the fixed set of an elliptic transformation is a $k$-dimensional hyperbolic plane, $0 \leq k \leq n-2$; thus providing the fixed point set with a geometric neighbourhood - together with its natural fat triangulation. Some complications may arise because different elliptics may well have fixed loci of different dimensions (see $\mathrm{Ap}$ ), so the respective simplices will have different dimensions and fatness. However this is easy to remedy by completing them to $n$-dimensional simplices, by 'expanding' the low dimensional neighbourhoods to maximal dimension in a product manner (see [S1] for details).

\section{ACKNOWLEDGMENT}

The author wishes to express his gratitude to Professor Uri Srebro for raising the problem and for his advice and assistance, and to Professors Robert Brooks and Bill Abikoff, for their support and many stimulating discussions. The author would also like to thank the referee for bringing to his attention the work of V. Mayer, as well as for his/her patience and many helpful comments.

\section{REFERENCES}

[Ab] W. Abikoff, Kleinian Groups, lecture notes, The Technion-Israel Institute of Technology, Haifa, Israel, 1996-1997.

[Al] J.W. Alexander, Note on Riemmann spaces, Bull. Amer. Math. Soc. 26 (1920), 370-372.

[Ap] B.N. Apanasov, Klein Groups in Space, Sib. Math. J. 16 (1975), 679-684. MR0404474 $(53: 8276)$

[Bea] A.F. Beardon, The Geometry of Discrete Groups, Springer Verlag, GTM 91, NY, 1982. MR.1393195 (97d:22011)

[Ber] M. Berger, Geometry II, Translated from the French by M. Cole and S. Levy. Universitext, Springer-Verlag, Berlin, 1987. MR0882916 (88a:51001b)

[BM] B.H. Bowditch and G. Mess, A 4-Dimensional Kleinian Group, Transaction of the Amer. Math. Soc. 344 (1994), no. 1, 390-405. MR1240944 (95f:57057)

[BrM] R. Brooks and J.P. Matelski, Collars in Kleinian groups, Duke Math. J. 49(1) (1982), 163-182. MR0650375 (83f:30039)

[Ca1] S.S. Cairns, On the triangulation of regular loci, Ann. of Math. 35 (1934), 579-587. MR.1503181

[Ca2] S.S. Cairns, Polyhedral approximation to regular loci, Ann. of Math. 37 (1936), 409-419. MR 1503287

[Ca3] S.S. Cairns, A simple triangulation method for smooth manifolds, Bull. Amer. Math. Soc. 67 (1961), 380-390. MR0149491 (26:6978) 
[CMS] J. Cheeger, W. Müller, and R. Schrader, On the Curvature of Piecewise Flat Spaces, Comm. Math. Phys. 92 (1984), 405-454. MR0734226 (85m:53037)

[Cox] H.S.M. Coxeter, Regular Polytopes, Second Edition, Macmillan, NY, 1963. MR0151873 $(27: 1856)$

[DM] D.A. Derevin and A.D. Mednikh, Geometric properties of discrete groups acting with fixed points in Lobachevsky space, Soviet Math. Dokl., 37(3) (1988), 614-617. MR0948799 (90a:30131)

[FM] M. Feighn and G. Mess, Conjugacy classes of finite subgroups of Kleinian groups, Amer. J. of Math. 113 (1991), 179-188. MR.1087807 (92a:57042)

[GM1] F.W. Gehring and G.J. Martin, Commutators, collars and the geometry of Möbius groups, J. Anal. Math. 63 (1994), 174-219. MR1269219 (96c:30040)

[GM2] F.W. Gehring and G.J. Martin, On the Margulis constant for Kleinian groups, I, Ann. Acad. Sci. Fenn. 21 (1996), 439-462. MR.1404096 (97f:30065)

[GMMR] F.W. Gehring, C. Maclachlan, G.J. Martin, and A.W. Reed, Arithmeticity, Discreteness and Volume, Trans. Amer. Math. Soc. 349 (1997), 3611-3643. MR1433117 (98d:57022)

[H] E. Hamilton, Geometrical finiteness for hyperbolic orbifolds, Topology 37(3) (1998), 635-657. MR:1604903 (99h:57027)

[Hu] J.F.P. Hudson, Piecewise Linear Topology, Math. Lect. Notes Series, Benjamin, NY, 1969. MR0248844(40:2094)

[J] T. Jørgensen, On discrete groups of Möbius transformations, Amer. J. of Math. 98(3) (1976), 739-749. MR0427627 (55:658)

[KP] M.E. Kapovitch and L. Potyagailo, On the Absence of Ahlfors and Sullivan theorems for Kleinian groups in higher dimensions, Sib. Math. J., Vol. 32, No. 1, 1991, pp. 227-237. MR 1138441 (93g:30064)

[KP1] M.E. Kapovitch and L. Potyagailo, On the absence of Ahlfors' finiteness theorem for Kleinian groups in dimension 3, Topology Appl. 40, 1991, pp. 83-91. MR1114093 (92j:57023)

[Med] A.D. Mednikh, Automorphism groups of the three-dimensional hyperbolic manifolds, Soviet Math. Dokl. 32(3) (1985), 633-636.

[Mor] J.W. Morgan, On Thurston's Uniformization Theorem for Three-Dimensional Manifolds, in The Smith Conjecture (Morgan, J.W. and Bass, H. ed.), Academic Press, NY, 1984, 37-126.

[MS1] O. Martio and U. Srebro, Automorphic quasimeromorphic mappings in $R^{n}$, Acta Math. 195 (1975), 221-247. MR0435388 (55:8348)

[MS2] O. Martio and U. Srebro, On the existence of automorphic quasimeromorphic mappings in $R^{n}$, Ann. Acad. Sci. Fenn., Series I Math. 3 (1977), 123-130. MR0585312 (58:28486)

[Ms] B. Maskit, Kleinian Groups, Springer Verlag, GDM 287, NY, 1987. MR0959135 (90a:30132)

[My] V. Mayer, Uniformly Quasiregular Mappings of Lattès Type, Conformal Geometry and Dynamics 1 (1997), 104-111. MR.1482944 (98j:30017)

[Mun] J.R. Munkres, Elementary Differential Topology (rev. ed.) Princeton University Press, Princeton, NJ, 1966. MR0198479 (33:6637)

[NW] P.J. Nicholls and P.L. Waterman, The boundary of convex fundamental domains for Fuchsian groups, Ann. Acad. Sci. Fenn., Ser A I Math. 15(1) (1990), 1-25. MR.1050778 (91h:30066)

[Pe] K. Peltonen, On the existence of quasiregular mappings, Ann. Acad. Sci. Fenn., Series I Math., Dissertationes, 1992. MR1165363(93h:30031)

[Rat] J.G. Ratcliffe, Foundations of Hyperbolic Manifolds, GTM 194, Springer Verlag, NY, 1994. MR:1299730 (95j:57011)

[S1] E. Saucan, The Existence of Quasimeromorphic Mappings, Ann. Acad. Sci. Fenn., Ser A I Math, 31, (2006), 131-142.

[S2] E. Saucan, Note on a theorem of Munkres, Mediterr. j. math 2(2) (2005), 215-229.

[S3] E. Saucan, in preparation.

[Som] D.M.Y. Sommerville, An Introduction to the Geometry of $N$ Dimensions, Dover Publications, NY, 1958. MR0100239 (20:6672)

[Spi V] M. Spivak, A Comprehensive Introduction to Differential Geometry, Vol. V, Publish or Perish, Boston, MA, 1975. MR0394453 (52:15254b) 
[Sr] U. Srebro, Non-existence of Automorphic Quasimeromorphic Mappings, Analysis and Topology, World Sci. Publishing, River Edge, NJ, 1998. MR.1667838 (99j:30027)

[SA] E. Saucan and E. Apleboim, Quasiconformal Fold Elimination for Seaming and Tomography, in preparation.

[Th] W. Thurston, Three-Dimensional Geometry and Topology, Vol. 1, (S. Levy, ed.), Princeton University Press, Princeton, NJ, 1997. MR1435975 (97m:57016)

[Tu] P. Tukia, Automorphic Quasimeromorphic Mappings for Torsionless Hyperbolic Groups, Ann. Acad. Sci. Fenn. 10 (1985), 545-560. MR0802519 (86k:30023)

[V] J. Väisalä, Lectures on n-dimensional quasiconformal mappings, Lecture Notes in Mathematics 229, Springer-Verlag, Berlin-Heidelberg-New-York, 1971. MR0454009 $(56: 12260)$

[Wh] J.H.C. Whitehead, On $\mathcal{C}^{1}$-complexes, Ann. of Math. 41 (1940), 809-824. MR0002545 $(2: 73 \mathrm{~d})$

Departments of Mathematics and Electrical Engineering, Technion, Haifa, Israel

E-mail address: semil@tx.technion.ac.il

E-mail address: semil@ee.technion.ac.il 